\title{
O Papel da Ocitocina na Profilaxia da Hemorragia Pós-Parto em Locais com Recursos Limitados
}

\section{The Role of Oxytocin in the Prevention of Postpartum Hemorrhage in Low-Resource Settings}

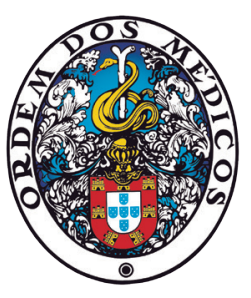

Inês FERREIRA $\triangle 1$, Ana REYNOLDS ${ }^{2,3,4}$

Acta Med Port 2021 Dec;34(12):857-863 - https://doi.org/10.20344/amp.14258

\section{RESUMO}

Introdução: A hemorragia pós-parto mantém-se como uma das principais causas de morte materna a nível global. A ocitocina é o uterotónico de eleição na profilaxia desta complicação. Contudo, a sua utilização em locais com recursos limitados associa-se a constrangimentos de foro clínico, político, económico e cultural. O objetivo deste artigo é rever o uso da ocitocina na profilaxia da hemorragia pós-parto em locais com recursos limitados.

Material e Métodos: Foi efetuada uma revisão da literatura sobre o tema, selecionando-se 24 artigos.

Resultados: A informação foi organizada em sete secções: a avaliação da eficácia da ocitocina relativamente a outros uterotónicos, a utilização da ocitocina em partos domiciliários, a capacitação dos profissionais de saúde, a qualidade da ocitocina disponibilizada nestes locais, as novas formulações, os riscos inerentes à utilização de uterotónicos e as políticas de saúde adotadas.

Discussão: Apesar dos progressos alcançados, verificámos que o acesso à profilaxia da hemorragia pós-parto com ocitocina em locais com recursos limitados está aquém do desejável. As principais dificuldades encontradas foram o défice de profissionais de saúde qualificados na administração da ocitocina, as deficiências na qualidade do fármaco e a desadequação das normas de orientação clínica existentes.

Conclusão: Para reduzir a mortalidade materna por hemorragia pós-parto em locais de recursos limitados é imprescindível melhorar a capacitação dos profissionais, implementar boas práticas no uso de uterotónicos, otimizar a gestão de recursos e transpor as barreiras culturais impeditivas da procura dos serviços de saúde.

Palavras-chave: Disparidades dos Cuidados de Saúde; Hemorragia Pós-Parto; Ocitocina; Países em Desenvolvimento; Saúde Materna

\section{ABSTRACT}

Introduction: Postpartum hemorrhage remains one of the leading causes of maternal death globally. Oxytocin is the uterotonic agent of choice for the prophylaxis of this complication. However, its use in low-resource settings is associated with clinical, political, economic and cultural constraints. The goal of this article is to describe the use of oxytocin for postpartum hemorrhage prophylaxis in low-resource settings.

Material and Methods: A literature review on the topic was carried out, and 24 articles were included.

Results: The information was organized into seven sections: the evaluation of the efficacy of oxytocin compared to other uterotonics, the use of oxytocin in home births, the training of healthcare professionals, the quality of the available oxytocin, the new formulations, the risks associated with the use of uterotonic and the adopted health policies.

Discussion: Despite the progress achieved widespread access to oxytocin for postpartum hemorrhage prophylaxis in low-resource settings is less than desirable. The main difficulties encountered were the shortage of skilled healthcare professionals for oxytocin administration, deficiencies concerning the quality of the drug and the inadequacy of available clinical guidelines.

Conclusion: In order to reduce maternal mortality caused by postpartum hemorrhage in low-resource settings, it is essential to improve the knowledge of healthcare professionals, to implement good practices on the use of uterotonics, to optimize resource management and to overcome cultural barriers that prevent the demand for health services.

Keywords: Developing Countries; Healthcare Disparities; Maternal Health; Oxytocin; Postpartum Hemorrhage

\section{INTRODUÇÃO}

Apesar de permanecer elevada, nos primeiros dezassete anos do século XXI, a taxa de mortalidade materna reduziu-se em 38\%. ${ }^{1}$ O terceiro objetivo do desenvolvimento sustentável da Organização das Nações Unidas visa a redução desta taxa, que em 2017 era de 211 por 100000 nados-vivos, para menos de 70 por 100000 nados-vivos, e que nenhum país isoladamente tenha mais que o dobro desta média mundial. ${ }^{1,2} \mathrm{Em} \mathrm{2017}$, aproximadamente 295000 mulheres morreram durante a gravidez, o parto e o pós-parto e quase todas estas mortes (94\%) ocorreram em locais com recursos limitados (LRL), sendo que a maioria poderia ter sido prevenida. ${ }^{1}$ Nestes locais verifica-se uma escassez de profissionais de saúde pelo que metade dos partos não são assistidos por profissionais qualificados. ${ }^{3}$

A hemorragia pós-parto (HPP) é a causa subjacente a um quarto das mortes maternas a nível global. ${ }^{4}$ É definida pela Organização Mundial de Saúde (OMS) como a perda de sangue igual ou superior a $500 \mathrm{~mL}$ nas 24 horas após

1. Mestrado Integrado em Medicina. Faculdade de Medicina. Universidade do Porto. Porto. Portugal.

2. Departamento de Ginecologia-Obstetrícia e Pediatria. Faculdade de Medicina. Universidade do Porto. Porto. Portugal.

3. Centro de Investigação em Tecnologias e Serviços de Saúde. Porto. Portugal.

4. Centro de Simulação Médica do Porto. São Mamede Infesta. Portugal.

$\triangle$ Autor correspondente: Inês Ferreira. inespocasferreira@gmail.com

Recebido: 02 de junho de 2020 - Aceite: 09 de novembro de 2020 - First published: 26 de fevereiro de 2021 - Online issue published: 02 de dezembro de 2021 Copyright $(\odot)$ Ordem dos Médicos 2021 
o parto. Quando a perda é igual ou superior a $1000 \mathrm{~mL}$ classifica-se como HPP grave. ${ }^{4}$

A gestão ativa do terceiro estadio do trabalho de parto engloba um conjunto de intervenções que procura prevenir a HPP. ${ }^{5}$ Inclui-se nestas a administração de uterotónico à parturiente até um minuto após o nascimento, conforme as normas da OMS. O uterotónico poderá ser a ocitocina (10 unidades internacionais (UI) por via intramuscular (IM) ou intravenosa (IV)), a carbetocina (100 $\mu \mathrm{g} \mathrm{IM} \mathrm{ou} \mathrm{IV),} \mathrm{o} \mathrm{miso-}$ prostol [400 $\mu \mathrm{g}$ ou $600 \mu \mathrm{g}$ per os (PO)], a ergometrina ou a metilergometrina (200 $\mu \mathrm{g}$ IM ou IV) ou a associação de ocitocina $5 \mathrm{UI}$ e ergometrina $500 \mu \mathrm{g}$ (IM). ${ }^{6}$ A ocitocina requer um profissional qualificado em administração de injetáveis, é relativamente barata, bem tolerada e está amplamente disponível no mercado, mas precisa de transporte e armazenamento refrigerados (entre $2^{\circ}$ a $8^{\circ} \mathrm{C}$ ), como a ergometrina. ${ }^{6,7}$ Quando estas condições não são asseguradas, devem considerar-se outros uterotónicos. O misoprostol e a carbetocina têm como principal vantagem a estabilidade à temperatura ambiente, mas só o misoprostol é administrável $\mathrm{PO}$, podendo ser utilizado por profissionais menos qualificados e nos partos domiciliários. , $^{6}$

A ocitocina é o fármaco de eleição na profilaxia e no tratamento da HPP, tendo também indicação na indução ou aceleração do trabalho de parto. ${ }^{8}$

Nos LRL há carência de meios para assegurar os cuidados de saúde a vários níveis, tais como, recursos humanos, materiais e infraestruturais. ${ }^{9}$ Isto relaciona-se com o baixo nível económico e a instabilidade política, pelo que se admite que os países de baixo e médio-baixo rendimento apresentam algum desequilíbrio destes recursos. Nos LRL descrevem-se três dificuldades na prestação dos cuidados de saúde à população, que são: o tardio reconhecimento da doença e da procura dos serviços de saúde, a demora no transporte até ao estabelecimento de saúde e a demora em receber os cuidados de saúde baseados na evidência. ${ }^{5}$

O objetivo deste artigo é rever o uso da ocitocina na profilaxia da HPP em LRL.

\section{MATERIAL E MÉTODOS}

Efetuámos uma análise descritiva da revisão dos artigos publicados sobre o uso da ocitocina para profilaxia da HPP em LRL. Foi efetuada uma pesquisa na PubMed a 5 de novembro de 2019. A query usada e a metodologia de pesquisa e seleção das publicações estão descritas na Fig. 1. Não se aplicaram restrições com base na data de publicação ou no idioma dos trabalhos publicados.

Analisámos ainda artigos potencialmente relevantes citados nas publicações obtidas. A seleção dos artigos foi feita por um observador, tanto pela leitura dos títulos como dos respetivos resumos, cada uma em duas datas diferentes. Leram-se integralmente os artigos selecionados, incluindo sete provenientes das listas de referências. Os critérios de inclusão foram: i) contexto aplicável a LRL; ii) nos estudos intervencionais, administração de ocitocina segundo as recomendações da OMS (10 UI de ocitocina IM ou IV); iii) parto por via vaginal. O contexto de LRL es- tabelecido incluiu o parto ocorrido em países de baixo ou médio-baixo rendimento, segundo os critérios de riqueza do Banco Mundial de 2019 - 2020. ${ }^{10}$

Os critérios de exclusão foram: i) estudos que comparam a eficácia dos agentes uterotónicos na prevenção da HPP que não incluíram a ocitocina ou são de revisão; ii) utilização de uterotónicos somente no tratamento da HPP; iii) artigos de opinião; iv) parto por cesariana; v) contexto não aplicável aos LRL; vi) cuidados de saúde exclusivamente no setor privado; vii) administração de ocitocina em dose diferente à recomendada pela OMS.

Não foi solicitado parecer à comissão de ética dado tratar-se duma revisão de dados publicados.

\section{RESULTADOS}

Através da leitura integral dos artigos selecionados permaneceram os 24 artigos que cumpriam os critérios de seleção (Fig. 1). A informação obtida foi organizada em sete tópicos, de acordo com a temática abordada.

\section{Ocitocina versus outros uterotónicos}

A variação dos níveis de hemoglobina num grupo de mulheres sujeitas a $400 \mu \mathrm{g}$ de misoprostol PO foi comparada com outro submetido a $10 \mathrm{UI}$ de ocitocina por via IM em dois ensaios clínicos duplamente cegos, randomizados e controlados com placebo. Um, entre 1998 e 1999, envolveu 392 parturientes num centro de referência hospitalar do Gana ${ }^{11}$ e outro com 499 mulheres num hospital terciário do Zimbabué, em 2001. ${ }^{12}$ Ambos concluíram que em muIheres de baixo risco a toma de misoprostol é tão eficaz na diminuição da perda hemorrágica no terceiro estádio do trabalho de parto quanto a de ocitocina IM e que o tremor e a pirexia foram efeitos adversos exclusivos do misoprostol. ${ }^{11,12} \mathrm{Em} \mathrm{2006}$, um estudo prospetivo randomizado e controlado $^{13}$ semelhante aos anteriores, que incluiu 2023 parturientes na Índia, comparou três uterotónicos, acrescentando um terceiro grupo que recebeu $2 \mathrm{mg}$ de ergometrina IV. Os autores constataram que o misoprostol é bem tolerado e tão eficaz quanto os restantes uterotónicos. ${ }^{13}$ Os efeitos adversos do misoprostol foram autolimitados. ${ }^{13}$ Ainda na Índia, entre 2010 e 2011, decorreu outro estudo prospetivo e randomizado com 300 mulheres $^{14}$ que comparou a administração de $600 \mu \mathrm{g}$ de misoprostol PO versus $10 \mathrm{UI}$ de ocitocina IM. O volume médio da perda hemorrágica, a mediana da descida da hemoglobina e a duração média da dequitadura não foram significativamente diferentes. ${ }^{14}$

Pela análise laboratorial ${ }^{15}$ dum análogo da ocitocina estável ao calor, a carbetocina, constatou-se que esta não é sensível à luz ou ao congelamento e que é comprovadamente estável na zona climática IV da International Conference of Harmonization $\left(30^{\circ} \mathrm{C}\right.$ e $75 \%$ de humidade relativa) por, pelo menos, três anos e por curtos períodos de tempo a temperaturas extremas. ${ }^{15}$ Num ensaio clínico randomizado, duplamente cego, de não-inferioridade, ${ }^{16}$ decorrido em 10 países entre 2015 e 2018 com 29645 parturientes, verificou-se que $100 \mu \mathrm{g}$ de carbetocina IM não têm eficácia inferior à da ocitocina na prevenção da HPP ligeira. ${ }^{16}$ 


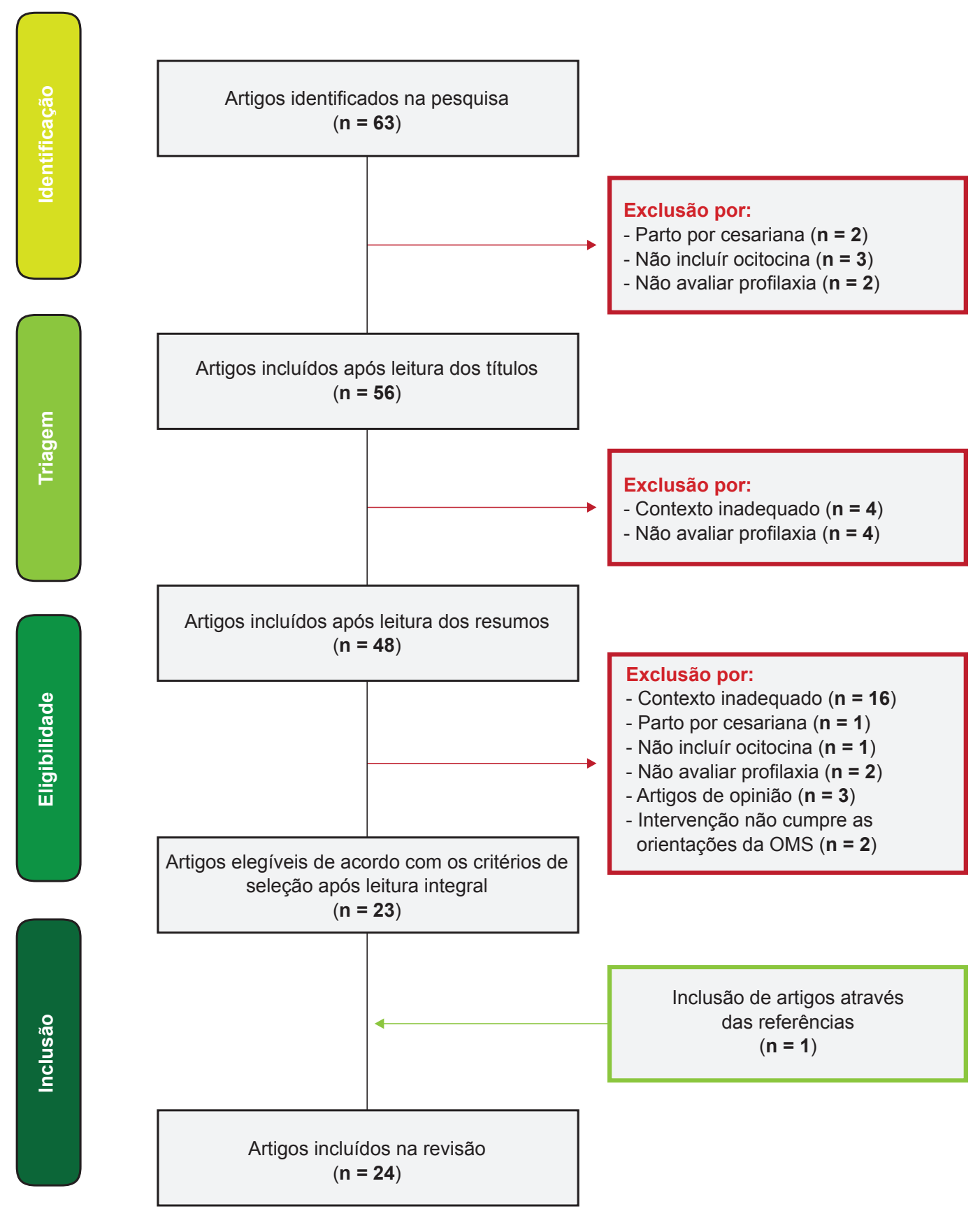

\footnotetext{
Query utilizada:

((“Oxytocin"[Mesh] OR "oxytocin"[All Fields]) AND (((“postpartum haemorrhage"[All Fields] OR "postpartum hemorrhage”[MeSH Terms] OR (“postpartum”[All Fields] AND "hemorrhage"[All Fields]) OR "Postpartum hemorrhage"[All Fields]) AND ("prevention and control"[Subheading] OR ("prevention"[All Fields] AND "control“[ALL Fields]) OR "prevention and control"[ALL Fields] OR "prevention"[ALL Fields]) OR "Postpartum Hemorrhage/prevention and control"[Mesh])) AND (“Poverty"[Mesh] OR "poverty"[All Fields] OR "low income" OR "middle income" OR "developing countr" OR "Poverty Areas"[Mesh] OR "International Cooperation"[Mesh] OR "Health Services Accessibility"[Mesh] OR "Healthcare Disparities"[Mesh] OR "Health Resources"[Mesh] OR "Limited-ResourceSettin" OR "Task-Sharing” OR "Task-Shifting" OR “Poor-Resouce Settin*” OR "Low-Resource Settin*” OR “Lower-middle Income Countr” OR (low-resource[All Fields] AND settings[All Fields]))
}

Figura 1 - Diagrama de fluxo: metodologia de pesquisa e seleção das publicações

OMS: Organização Mundial de Saúde

Todavia, não foi possível comprovar o mesmo relativamente à HPP grave pois a sua taxa de ocorrência foi baixa.

Não se constataram diferenças relativas a efeitos adversos ou à necessidade de intervenções adicionais. ${ }^{16}$

Duas análises de custo-eficácia indicaram coortes hipotéticas com 1000 e 150000 mulheres, uma em ambiente misto (40\% dos nascimentos no hospital e $60 \%$ na comunidade $)^{9}$ e outra na comunidade em zonas rurais do Senegal, ${ }^{17}$ respetivamente. Os autores concluíram que o misoprostol, comparativamente à ocitocina e à não 
administração de uterotónico, é capaz de reduzir significativamente custos e melhorar o desfecho materno em LRL, onde a ocitocina é inacessível. ${ }^{9,17}$

\section{Ocitocina em partos domiciliários ou na comunidade}

No Gana, entre 2011 e 2012, realizou-se um ensaio clínico randomizado em clusters, com 1586 parturientes. ${ }^{18}$ Neste concluiu-se que a administração de 10 UI de ocitocina por via IM para profilaxia da HPP em partos domiciliários, realizada por agentes de saúde comunitária, sem formação em cuidados obstétricos, é eficaz, segura e viável. ${ }^{18}$ Não foram reportados casos de uso indevido da ocitocina, isto é, não foi administrada antes do terceiro estádio do trabalho de parto. ${ }^{18}$ Contudo, surgiram preocupações como o desconhecimento da técnica de injeção, a administração de ocitocina antes do nascimento, e a labilidade térmica da ocitocina. $^{18}$

\section{Capacitação em saúde para a utilização de ocitocina}

Numa zona rural das Honduras avaliaram-se 568 partos entre 2004 e 2008, onde assistentes de enfermagem receberam treino para a administração de ocitocina IM. ${ }^{19}$ Após a intervenção, a administração atempada de ocitocina aumentou de $63,8 \%$ para $96,5 \%$ e a taxa de HPP reduziu significativamente, de $14,8 \%$ para $5,9 \%{ }^{19}$ Porém, o uso de ocitocina antes do terceiro estadio também aumentou significativamente de $6,1 \%$ para $22,7 \%{ }^{19}$

Na Tanzânia, o Ministério da Saúde desenvolveu intervenções para melhorar a prevenção da HPP. Antes e após as mesmas, um estudo transversal acompanhou 52 instituições de saúde, em 2010 e 2012. ${ }^{20}$ O uso profilático de ocitocina subiu $37,1 \%$ em centros de saúde e dispensários, mas manteve-se nos hospitais. ${ }^{20}$ Foi reportado que a disponibilidade global da ocitocina subiu de $73 \%$ para $94 \%$ e que as instalações de nível mais básico têm menor quantidade e rotatividade dos profissionais de saúde que os hospitais..$^{20}$

Entre 2015 e 2016, um estudo qualitativo decorrido na Etiópia, Índia e Mianmar, ${ }^{21}$ através de observação direta e da aplicação de inquéritos à comunidade, constatou que, entre 198 profissionais de saúde, decisores políticos e especialistas de cadeias de transporte, há desconhecimento sobre a sensibilidade térmica da ocitocina e os efeitos do transporte na qualidade do fármaco. ${ }^{21}$ Neste âmbito, um estudo transversal descritivo de 2017 avaliou por questionário a experiência clínica relativa à qualidade da ocitocina na Nigéria. ${ }^{22}$ Apenas $52 \%$ dos 705 inquiridos sabia que a ocitocina deve ser armazenada a $2^{\circ}-8^{\circ} \mathrm{C}, 80 \%$ usa o fármaco para acelerar e $68 \%$ para induzir o trabalho de parto, e $13 \%$ acredita que já utilizou ocitocina ineficaz. Contudo somente $36 \%$ tem meios para o documentar, e $51 \%$ usa 20 ou mais UI de ocitocina na profilaxia da HPP. ${ }^{22}$

\section{Qualidade da ocitocina disponível em LRL}

Numa revisão sistemática ${ }^{23}$ que englobou 559 amostras de ocitocina de 15 países de LRL e num estudo exploratório ${ }^{24}$ que analisou 256 amostras da República Democrática do Congo em 2016, verificou-se que nos LRL há uma eleva- da prevalência de ampolas de ocitocina de baixa qualidade que é, presumivelmente, devida a problemas de fabrico e ao deficiente armazenamento. Na revisão, duas amostras não tinham o ingrediente ativo. ${ }^{23}$ Nesta, mais de um terço das amostras, e no estudo exploratório, mais de $80 \%$, apresentavam teor de ocitocina inferior a $90 \% .{ }^{23,24}$ No último, as amostras continham, em média, um nível de impureza de $12,3 \%$, mas nenhuma falhou o teste de esterilidade. ${ }^{24}$

O estudo observacional decorrido no Gana em 2015 mostrou que a atividade da ocitocina não foi afetada pela cadeia de distribuição durante um mês, tendo as 130 amostras sido sujeitas a temperaturas entre $-9,9^{\circ} \mathrm{C}$ e $+30,1^{\circ} \mathrm{C} .^{25}$

\section{Perspetivas futuras em relação à ocitocina}

Duas novas formulações da ocitocina com menor labilidade térmica encontram-se em estudo.

$\mathrm{Na}$ Austrália, desenvolveu-se a ocitocina inalatória, em aerossol para absorção pulmonar. Através do estudo de efeito na atividade uterina, in vitro e em ovelhas pós-parto, foi demonstrado o seu potencial uterotónico. ${ }^{26}$ Entre 2015 e 2016, através dum inquérito com discussões em grupos focais e entrevistas em profundidade, os autores constataram que esta é viável e aceitável na comunidade do Mianmar, onde os partos domiciliários são frequentes e culturalmente preferidos, embora haja preocupação com o uso inadequado. ${ }^{27}$ Os autores realçam que esta via depende da colaboração da parturiente e que se encontra em estudo para uso na profilaxia da HPP. ${ }^{27}$

Nos Estados Unidos da América surgiu a formulação sublingual de ocitocina liofilizada, um comprimido de dissolução rápida que se mantém estável entre $40^{\circ} \mathrm{C}$ e $75 \%$ de humidade relativa durante 12 meses. ${ }^{28} \mathrm{~A}$ avaliação in vitro e em porcos miniatura do lucatão demonstrou que a concentração plasmática máxima de ocitocina atingida foi menor que a obtida através da injeção IM de ocitocina (207 versus $612 \mathrm{pg} / \mathrm{mL}$, respetivamente). ${ }^{28}$

\section{Riscos da disponibilização de uterotónicos em LRL}

Uma revisão da literatura ${ }^{29}$ de 2007 concluiu que a disponibilidade de uterotónicos em países de baixo rendimento associa-se a taxas de indução e aceleração do trabaIho de parto até próximo de $50 \%$ dos partos hospitalares. Apesar da escassez de dados, os autores sugerem uma relação direta entre esta prática e o aumento do número de casos com necessidade de reanimação neonatal, de nados-mortos e de rotura uterina. ${ }^{29}$

No Uganda, através de entrevistas a 82 informadores-chave e da análise da introdução do misoprostol no país verificada em documentos governamentais e de aquisição de fármacos, constatou-se que, entre 2010 e 2013, cinco Organizações da Sociedade Civil forçaram a introdução e a utilização de misoprostol nos serviços de saúde com capacidade para usar ocitocina, e promoveram o seu uso em indicações não aprovadas. ${ }^{30}$

\section{Política e diplomacia}

Em 2006, a ação conjunta da International Federation 
of Gynecology and Obstetrics e da International Confederation of Midwives descreveu 10 ações chave para prevenir e tratar a HPP. ${ }^{5}$ As ações preventivas baseiam-se em i) obter apoio e disseminar informação por meios governamentais, sociais, formativos e clínicos; ii) garantir que todas as parturientes têm acesso à gestão ativa do terceiro estadio do trabalho de parto e que os profissionais de saúde estão capacitados; iii) garantir equipamento, fármacos e protocolos de atuação nos locais de parto; iv) avaliar novas tecnologias e fármacos que visem a prevenção da HPP. ${ }^{5}$

Através de um questionário aplicado a órgãos governamentais de 37 países, ${ }^{31}$ constatou-se que $89 \%$ reportou disponibilidade regular de ocitocina nas instituições de saúde. Em 26 países (70\%) a ocitocina é distribuída gratuitamente, mas em nove destes (35\%), os doentes pagam, por vezes, pelo fármaco. Em todos os países, exceto na Guiné Equatorial, a ocitocina consta da lista de medicamentos essenciais para a prevenção da HPP. A ocitocina integra a gestão ativa do terceiro estádio do trabalho de parto nas 20 normas nacionais analisadas, mas apenas 18 indicam a dose correta e somente nove descrevem adequadamente os procedimentos dessa gestão. ${ }^{31}$

Na Etiópia, em 2016, inquiriram-se 244 membros e profissionais da comunidade através de discussões em grupos focais e entrevistas em profundidade, explorando-se a possível introdução da ocitocina inalatória. ${ }^{32} \mathrm{~A}$ aprovação por órgãos rigorosos, como a OMS, foi apontada como um facilitador essencial da aprovação regulatória a nível nacional. Os compradores expressaram elevada sensibilidade ao preço, mas estavam dispostos a pagar por uma formulação mais eficaz. O requisito de compra direta a um único fabricante era uma potencial barreira. ${ }^{32}$

O estudo decorrido no Uganda, ${ }^{30}$ descrito anteriormente, demonstrou que o envolvimento direto de organizações da sociedade civil acelerou o processo de registo do misoprostol pelas autoridades nacionais reguladoras de medicamentos e a elaboração de normas clínicas. Inclusivamente, estas organizações, em colaboração com fornecedores nacionais, adquiriram e distribuíram misoprostol pelos centros de saúde dois anos antes da inclusão deste fármaco nas normas de orientação clínica e na ausência de evidência. $^{30}$

\section{DISCUSSÃO}

Atualmente, a HPP é uma causa de morte materna prevenível. Contudo, a sua mitigação em LRL é um problema de difícil resolução. Apesar dos progressos conseguidos nos últimos anos, continua a ser uma das principais causas de óbito materno. ${ }^{1}$

Globalmente, dos uterotónicos disponíveis para profilaxia da HPP, a ocitocina é o fármaco de eleição. Porém, nos LRL, o misoprostol tem ganho destaque dado apresentar melhor custo-eficácia, não exigir condições térmicas específicas, e ser administrável por agentes menos qualificados, embora o tremor e a pirexia sejam efeitos adversos frequentes. 9,11-14,17 A carbetocina tem eficácia sobreponível à ocitocina na profilaxia da HPP ligeira e permanece es- tável a temperaturas elevadas. ${ }^{15,16}$ Embora apresente perfil semelhante de efeitos adversos à ocitocina, também é injetável exigindo assistência qualificada. As formulações de ocitocina em desenvolvimento, sublingual e inalatória, demonstraram resultados promissores mas encontram-se, ainda, em estudo. ${ }^{26-28}$

Assim, caso a ocitocina esteja indisponível, na suspeita de má qualidade ou inexistência de pessoal qualificado para a administrar, as recomendações da OMS de $2018^{6}$ incluíram o uso do misoprostol e da carbetocina na profilaxia da HPP.

Nos LRL, o parto ocorre usualmente no domicílio sem assistência por um profissional de saúde qualificado. Deste modo, o uso generalizado da ocitocina é de difícil implementação. A possibilidade da sua administração fora dos estabelecimentos de saúde por agentes de saúde comunitária é uma hipótese que carece de reavaliação. ${ }^{18}$

Relativamente aos profissionais de saúde, torna-se evidente a necessidade de melhorar as aptidões da assistência ao parto, sobretudo daqueles que trabalham em centros de saúde mais isolados e com escassos recursos. ${ }^{20}$ Esta capacitação implicou um aumento significativo da utilização atempada da ocitocina para profilaxia da HPP. ${ }^{19}$ Todavia, além de requerer o aprovisionamento do fármaco, constatou-se um aumento alarmante do seu uso indevido. ${ }^{19} \mathrm{~A}$ inexistência de normas de orientação clínica nos LRL e a falta de consciencialização para os potenciais efeitos nefastos do uso indevido dos uterotónicos são problemas a considerar aquando da sua disponibilização. ${ }^{21,22,31}$

A qualidade da ocitocina disponível nos LRL é outro desafio. A fraca qualidade deve-se à labilidade térmica do fármaco, à produção de má qualidade e à contrafação, levando comummente à administração de doses superiores às recomendadas para se obter o mesmo efeito. ${ }^{22-24} \mathrm{~A}$ situação agrava-se pelo desconhecimento generalizado e incapacidade no cumprimento das condições de armazenamento e transporte. ${ }^{21}$ A necessidade de formação e discussão deste assunto com profissionais de saúde, decisores políticos e membros da cadeia de distribuição é fulcral para se criarem normas de atuação relativas ao uso da ocitocina.

A importância da ocitocina na profilaxia da HPP é universalmente reconhecida. Porém, nos LRL, a escassez de recursos, a inexistência de indicadores de saúde materna e de sistemas de monitorização nacionais têm dificultado a implementação ou ampliação de intervenções. ${ }^{31}$ As normas de orientação clínica disponíveis estão frequentemente incompletas ou desatualizadas, sendo essencial mudar este paradigma. ${ }^{31}$ É necessário atender às diferenças entre os vários contextos e analisar a visão da parturiente e familiares.

Ainda a nível diplomático, é importante analisar o crescente papel das organizações da sociedade civil, tanto na promoção de boas práticas como de estratégias com efeitos potencialmente danosos. ${ }^{30}$

A revisão elaborada sem limites de pesquisa permitiu incluir várias nações e subconjuntos culturais, políticos e ambientais, possibilitando uma representação abrangente 
do conhecimento e práticas em torno do uso da ocitocina na profilaxia da HPP nos LRL. Contudo, entende-se que existem diferenças dentro de cada país e entre países do mesmo nível de rendimento, o que limita a extrapolação direta das conclusões além das regiões investigadas. A falta da análise formal da qualidade da informação de cada artigo é uma limitação, tal como a utilização de apenas uma base de dados.

O contraste no desfecho materno em situações de HPP entre os LRL e os demais locais expõe diferenças nos cuidados de saúde, permitindo apontar importantes constrangimentos verificados nos LRL, passíveis de ultrapassar.

\section{CONCLUSÃO}

A HPP é um problema de dimensões globais e com consequências graves. A ocitocina é o uterotónico de eleição na sua profilaxia, embora em LRL apresente importantes constrangimentos. A capacitação dos agentes de saúde no uso da ocitocina para profilaxia da HPP tem-se revelado profícua, mas não se pode negligenciar o possível uso indevido do fármaco.

Torna-se imprescindível investir numa abordagem diplomática que permita universalizar práticas e tornar a distribuição e a utilização da ocitocina mais rigorosas nos LRL. É, também, imperativo transpor barreiras culturais impeditivas da procura dos serviços de saúde pela população.

O conjunto de problemas encontrados não se circuns-

\section{REFERÊNCIAS}

1. World Health Organization. Trends in maternal mortality 2000 to 2017 estimates by WHO, UNICEF, UNFPA, World Bank Group and the United Nations Population Division. Geneva: WHO; 2019.

2. Unstats.un.org. New York: World Health Organization and United Nations Children's Fund joint database on SDG 3; c2019. [consultado 2020 fev 10]. Disponível em: https://unstats.un.org/sdgs/indicators/ database/.

3. Raams TM, Browne JL, Festen-Schrier V, Klipstein-Grobusch K, Rijken MJ. Task shifting in active management of the third stage of labor: a systematic review. BMC Pregnancy Childbirth. 2018;18:47.

4. World Health Organization. WHO recommendations for the prevention and treatment of postpartum haemorrhage [e-book]. $1^{\text {st }}$ ed; 2012. [consultado 2020 fev 2]. Disponível em: WHO sexual and reproductive health.

5. Lalonde A, Daviss BA, Acosta A, Herschderfer K. Postpartum hemorrhage today: ICM/FIGO initiative 2004-2006. Int J Gynaecol Obstet. 2006;94:243-53.

6. World Health Organization. WHO recommendations: uterotonics for the prevention of postpartum haemorrhage [e-book]. 2018. [consultado 2020 fev 02]. Disponível em: WHO Institutional Repository for Information Sharing.

7. World Health Organization. The international pharmacopoeia: monographs: dosage forms: specific monographs: oxytocin injection (Oxytocini injectio). 9 $9^{\text {th }}$ ed. Geneva: WHO; 2019.

8. Medicines.org.uk. Berkshire: Oxytocin $10 \mathrm{IU} / \mathrm{ml}$ Solution for infusion; c2015. [consultado 2020 jan 30]. Disponível em: https://www.medicines. org.uk/emc/medicine/30427.

9. Lang DL, Zhao FL, Robertson J. Prevention of postpartum haemorrhage: cost consequences analysis of misoprostol in low-resource settings. BMC Pregnancy Childbirth. 2015;15:305.

10. Datahelpdesk.worldbank.org. World Bank Country and Lending Groups c 2019. [consultado 2019 nov 01]. Disponível em: https://datahelpdesk. worldbank.org/knowledgebase/articles/906519-world-bank-countryand-lending-groups.

11. Walley RL, Wilson JB, Crane JM, Matthews K, Sawyer E, Hutchens D. A double-blind placebo controlled randomised trial of misoprostol creverá a esta situação clínica, podendo estender-se a outras condições. A abordagem holística no que respeita à assistência à saúde materna nos $L R L$ é da máxima relevância.

\section{CONTRIBUTO DOS AUTORES}

IF: Desenho, elaboração, análise de dados e redação do artigo.

AR: Desenho, interpretação de dados, redação e revisão crítica do artigo.

\section{PROTEÇÃO DE PESSOAS E ANIMAIS}

As autoras declaram que os procedimentos seguidos estavam de acordo com os regulamentos estabelecidos pelos responsáveis da Comissão de Investigação Clínica e Ética e de acordo com a Declaração de Helsínquia da Associação Médica Mundial atualizada em 2013.

\section{CONFIDENCIALIDADE DOS DADOS}

As autoras declaram ter seguido os protocolos do seu centro de formação acerca da publicação de dados.

\section{CONFLITOS DE INTERESSE}

As autoras declaram não ter conflitos de interesse.

\section{FONTES DE FINANCIAMENTO}

Não houve qualquer fonte de financiamento.

and oxytocin in the management of the third stage of labour. BJOG. 2000;107:1111-5.

12. Kundodyiwa TW, Majoko F, Rusakaniko S. Misoprostol versus oxytocin in the third stage of labor. Int J Gynaecol Obstet. 2001;75:235-41.

13. Zachariah ES, Naidu M, Seshadri L. Oral misoprostol in the third stage of labor. Int J Gynaecol Obstet. 2006;92:23-6.

14. Mukta M, Sahay PB. Role of misoprostol $600 \mathrm{mcg}$ oral in active management of third stage of labor: a comparative study with oxytocin 10 IU i.m. J Obstet Gynaecol India. 2013;63:325-7.

15. Malm M, Madsen I, Kjellstrom J. Development and stability of a heatstable formulation of carbetocin for the prevention of postpartum haemorrhage for use in low and middle-income countries. J Pept Sci. 2018;24:e3082

16. Widmer M, Piaggio G, Nguyen TM, Osoti A, Owa OO, Misra S, et al. Heat-stable carbetocin versus oxytocin to prevent hemorrhage after vaginal birth. N Engl J Med. 2018;379:743-52.

17. Vlassoff M, Diallo A, Philbin J, Kost K, Bankole A. Cost-effectiveness of two interventions for the prevention of postpartum hemorrhage in Senegal. Int J Gynaecol Obstet. 2016;133:307-11.

18. Stanton CK, Newton S, Mullany LC, Cofie P, Tawiah Agyemang C, Adiibokah $\mathrm{E}$, et al. Effect on postpartum hemorrhage of prophylactic oxytocin $(10 \mathrm{IU})$ by injection by community health officers in Ghana: a community-based, cluster-randomized trial. PLoS Med. 2013;10:e1001524.

19. Low LK, Bailey JM, Sacks E, Robles C, Medina L. Reduced postpartum hemorrhage after implementation of active management of the third stage of labor in rural Honduras. Int J Gynaecol Obstet. 2012;119:21720.

20. Bishanga DR, Charles J, Tibaijuka G, Mutayoba R, Drake M, Kim YM, et al. Improvement in the active management of the third stage of labor for the prevention of postpartum hemorrhage in Tanzania: a cross-sectional study. BMC Pregnancy Childbirth. 2018;18:223.

21. Oliver VL, Lambert PA, Than KK, Mohamed Y, Luchters S, Verma S, et al. Knowledge, perception and practice towards oxytocin stability and quality: a qualitative study of stakeholders in three resource-limited countries. PLoS One. 2018;13:e0203810. 
22. Ejekam CS, Okafor IP, Anyakora C, Ozomata EA, Okunade K, Oridota $\mathrm{SE}$, et al. Clinical experiences with the use of oxytocin injection by healthcare providers in a southwestern state of Nigeria: a crosssectional study. PLoS One. 2019;14:e0208367.

23. Torloni MR, Gomes Freitas C, Kartoglu UH, Metin Gulmezoglu A, Widmer M. Quality of oxytocin available in low- and middle-income countries: a systematic review of the literature. BJOG. 2016;123:207686.

24. Lambert P, Nguyen TH, McEvoy C, Minhas RS, Wright P, Deadman K, et al. Quality of oxytocin ampoules available in health care facilities in the Democratic Republic of Congo: an exploratory study in five provinces. J Glob Health. 2018;8:020415.

25. Kartoglu U, Widmer M, Gulmezoglu M. Stability of oxytocin along the supply chain: a WHO observational study. Biologicals. 2017;50:117-24.

26. Prankerd RJ, Nguyen TH, Ibrahim JP, Bischof RJ, Nassta GC, Olerile LD, et al. Pulmonary delivery of an ultra-fine oxytocin dry powder formulation: potential for treatment of postpartum haemorrhage in developing countries. PLoS One. 2013;8:e82965.

27. Than KK, Oliver V, Mohamed $Y$, La T, Lambert P, Mclntosh M, et al. Assessing the operational feasibility and acceptability of an inhalable formulation of oxytocin for improving community-based prevention of postpartum haemorrhage in Myanmar: a qualitative inquiry. BMJ Open. 2018;8:e022140.

28. Zhu C, Estrada M, White J, Lal M. Heat-stable sublingual oxytocin tablets as a potential needle-free approach for preventing postpartum hemorrhage in low-resource settings. Drug Deliv Transl Res. 2018;8:853-6.

29. Lovold A, Stanton C, Armbruster D. How to avoid iatrogenic morbidity and mortality while increasing availability of oxytocin and misoprostol for PPH prevention? Int J Gynaecol Obstet. 2008;103:276-82.

30. Atukunda EC, Brhlikova P, Agaba AG, Pollock AM. Civil society organizations and medicines policy change: a case study of registration, procurement, distribution and use of misoprostol in Uganda. Soc Sci Med. 2015;130:242-9.

31. Smith JM, Currie S, Cannon T, Armbruster D, Perri J. Are national policies and programs for prevention and management of postpartum hemorrhage and preeclampsia adequate? A key informant survey in 37 countries. Glob Health Sci Pract. 2014;2:275-84.

32. Oliver V, Tolera M, Teklu AM, Minaye A, Lambert P, Mclntosh M. Qualitative formative implementation research to inform introduction of a new essential medicine. Res Social Adm Pharm. 2020;16:535-43. 